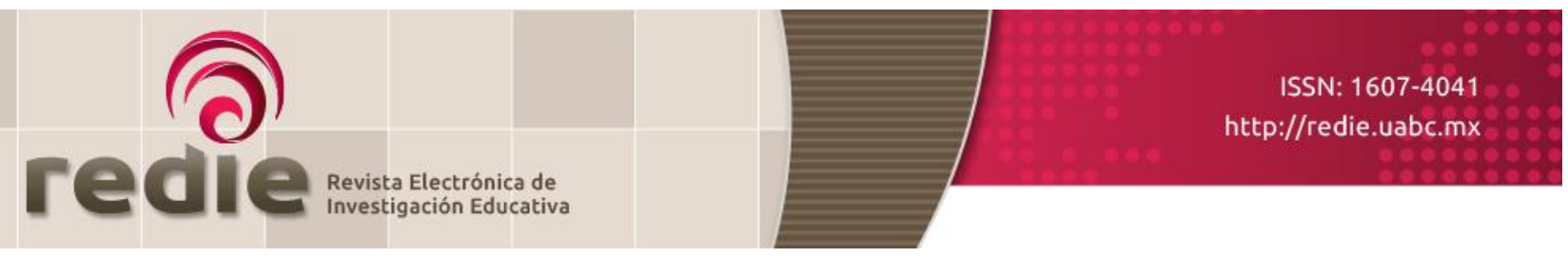

Vol. 19, Núm. 3, 2017

\title{
Aprendizajes, inclusión y justicia social en entornos educativos multiculturales
}

\section{Learning, Inclusion and Social Justice in Multicultural Educational Environments}

Felipe Jiménez Vargas (1) felipe.jimenez@ucv.cl

José Luis Lalueza Sazatornil (2) joseluis.lalueza@uab.cat

Carla Fardella Cisternas (3) fardellacarla@hotmail.com

(1) Pontificia Universidad Católica de Valparaíso

(2) Universidad Autónoma de Barcelona

(3) Universidad Andrés Bello

(Recibido: 25 de julio de 2014; Aceptado para su publicación: 5 de enero de 2016)

Cómo citar: Jiménez, F., Lalueza, J. L. y Fardella, K. (2017). Aprendizajes, inclusión y justicia social en entornos educativos multiculturales. Revista Electrónica de Investigación Educativa, 19(3), 10-23. https://doi.org/10.24320/redie.2017.19.3.830

\section{Resumen}

En el escenario educativo actual, marcado por el incremento sistemático de grupos minoritarios en las aulas, así como por las desigualdades que se reproducen y actualizan en los contextos educativos, el desarrollo de propuestas inspiradas en principios de inclusión, multiculturalidad y justicia social cobran cada vez más relevancia. Mediante un trabajo etnográfico de observación participante en una escuela pública del área metropolitana de Barcelona, esta investigación analiza dos sistemas de actividad educativa en un mismo contexto escolar: procesos de enseñanza-aprendizaje basados en enfoques tradicionales y procesos de enseñanza-aprendizaje inspirados en el modelo Quinta Dimensión (5D). Los resultados evidencian que cuando los sistemas de actividad permiten a sus participantes vincular los contenidos curriculares a sus referentes culturales, cuando se privilegian interacciones colaborativas orientadas a metas compartidas y se ajustan las ayudas educativas a las necesidades de los educando, se amplían las oportunidades de éxito escolar para y se eliminan barreras para su aprendizaje y participación.

Palabras clave: Multiculturalismo, educación inclusiva, justicia social, investigación educativa, etnografía escolar.

\section{Abstract}

In the current educational setting, marked by a systematic increase in minority groups in classrooms and inequalities that are reproduced and modernized in educational contexts, the development of educational proposals modeled on the principles of inclusion, multiculturalism and social justice garners ever greater prominence and academic interest. By way of an ethnographic observational study conducted in a public school in the metropolitan area of Barcelona, this research analyzes two educational activity systems in the same academic context: teaching-learning processes based on traditional approaches and teaching-learning processes modeled on the Fifth Dimension (5D). The results show that when activity systems allow participants to make a connection between curriculum content 
and their cultural references, when priority is given to collaborative interaction oriented toward shared goals, and when learning aids are adapted to participants' needs, all students' chances of academic success are substantially enhanced and obstacles to their learning and participation in school are erased.

Keywords: Multiculturalism, inclusive education, social justice, educational research, social ethnography.

\section{Introducción}

Investigaciones realizadas en los últimos años (Besalú, 2010; Garreta, 2011; Jiménez y Fardella, 2012) ofrecen un panorama general de lo que el fenómeno de la inmigración ha supuesto para las escuelas. En términos generales sus hallazgos y discusiones advierten de la complejidad y urgencia de las problemáticas que la escolarización del alumnado inmigrante y perteneciente a minorías étnicas ha supuesto para el sistema educativo en su conjunto, pero con mayor énfasis en las escuelas públicas, que es donde esta población se concentra.

Dentro del campo de estudio del fracaso escolar, Banks (1989), Lalueza (2012), Echeita y Sandoval (2002) y Ogbu (2005) han contribuido a complejizar las explicaciones tradicionales en torno al déficit cultural de los grupos minoritarios e inmigrantes, resaltando la influencia que las discontinuidades entre la cultura escolar y la cultura familiar tienen en el fracaso del alumnado inmigrante. De forma consensuada estos autores advierten de la dificultad, o incluso la imposibilidad de lograr el éxito escolar del alumnado minoritario, si la escuela continúa operando bajo una lógica monocultural, sin promover el despliegue de continuidades entre la cultura escolar y las culturas de adscripción y pertenencia de los estudiantes. En esa misma línea, estudios etnográficos realizados en el contexto español por Lamas y Lalueza (2012), Pàmies (2011) y Poveda (2003) ofrecen una aguda mirada al interior de las escuelas y aulas con alumnado minoritario o inmigrante. A través de sus hallazgos evidencian los riesgos que supone para éstos la participación en procesos de escolarización en los cuales los referentes culturales del alumnado no son considerados en el currículum escolar, generando profundas dificultades para conectar el currículum formal con sus conocimientos previos y sus fondos de conocimientos (Esteban-Guitart, Oller y Vila, 2012).

En este escenario, marcado por las profundas desigualdades educativas que afectan a estudiantes pertenecientes a grupos minoritarios (OECD, 2013), la presente investigación busca identificar y visibilizar prácticas educativas que contribuyen a reproducir dichas desigualdades, lo que se traduce finalmente en oportunidades de aprendizaje y participación escolar de menor calidad para determinados estudiantes. A su vez, y a través del análisis de un modelo inclusivo de innovación educativa, se aportan datos empíricos que sirvan para la discusión en torno al diseño de procesos de enseñanza y aprendizaje culturalmente pertinentes y socialmente justos (Connell, 1997).

En un primer apartado se sintetiza un conjunto de principios de la educación inclusiva y se caracteriza el modelo de la Quinta Dimensión (5D) implementado como sistema de actividad inclusivo. En un segundo momento se detallan las decisiones metodológicas tomadas para realizar la investigación y, finalmente, se presentan los principales hallazgos y la discusión en torno a ellos.

Aun cuando el movimiento por la inclusión educativa en contextos de diversidad cultural tiene una historia relativamente corta como modelo explicativo de la gestión de la diversidad en las escuelas (Jiménez y Fardella, 2015), ha ido ganando terreno de manera contundente tanto en el mundo académico como en el ámbito profesional. Prueba de ello es el progresivo y sistemático aumento que se evidencia en las publicaciones científicas a nivel mundial en materia de investigación educativa. Si bien son diversos los factores que contribuyen a explicar ambos fenómenos -mayor adherencia y aumento de publicaciones- a nuestro juicio uno de ellos radica en que el modelo de inclusión supone la superación de enfoques de gestión basados en la lógica de la segregación y la asimilación (Ainscow, 2013). Dicho de manera inversa, separar o aislar en escuelas especialmente diseñadas a un determinado tipo de estudiantes en función de sus capacidades, origen sociocultural, pertenencia étnica u otras variables, así como forzar su ajuste y adaptación a la cultura escolar dominante, no tiene otro efecto sino aumentar la brecha de desigualdad en los logros y las oportunidades en comparación al alumnado perteneciente a la cultura hegemónica. En ese sentido, los escenarios educativos multiculturales se han mostrado como un espacio privilegiado para evidenciar las raíces etnocéntricas y monoculturales de nuestros sistemas 
educativos, así como la contribución de éstos en el mantenimiento de las desigualdades sociales expresadas en la escuela (Lawrence-Brown y Sapon-Shevin, 2014).

Una idea central de la educación inclusiva consiste en asumir que las dificultades educativas por las que atraviesan ciertos estudiantes no radican en condiciones, personales e internas, que los hacen requerir atención educativa especial, sino más bien que las dificultades que enfrentan los estudiantes en su experiencia escolar corresponden a barreras para el aprendizaje y la participación que son instaladas implícita o explícitamente-, por el propio funcionamiento de la escuela y su cultura escolar (Booth y Ainscow, 2011). Desde una perspectiva ecológica de análisis (Bronfenbrenner, 1987) es posible identificar diversas modalidades de barreras que constriñen la inclusión educativa. Para los fines de este trabajo se presentan tres de ellas, que adquieren mayor relevancia en contextos educativos con una importante diversidad cultural.

Desde una perspectiva sociocultural de entender lo psicológico y su relación con los procesos de enseñanza y aprendizaje (Vigotsky, 1978), la naturaleza cultural y situada de los conocimientos previos de los estudiantes se constituyen en un elemento central de los aprendizajes. Situar estos conocimientos en un lugar privilegiado de los procesos de enseñanza y aprendizaje supone entenderlos como una unidad indisociable del patrimonio y herencia cultural de dichos estudiantes, sus familias y sus comunidades de base. El rol que estos fondos de conocimientos juegan dentro del aula es decisivo para la inclusión educativa (Esteban-Guitart y Moll, 2014), ya que no sólo permiten dar significatividad lógica y psicológica a los contenidos del currículum (Cöll, 1988), sino que otorgan, además, continuidad y pertinencia cultural a los aprendizajes (Cochran-Smith, Ludlow, O'Leary y Enterline 2012; Lazar, 2013). De esta manera, el reconocimiento de los fondos de conocimientos se constituye en una primera forma de eliminar barreras para el aprendizaje y la participación del alumnado, convirtiéndose, en consecuencia, en una primera pieza clave de un sistema de actividad educativo organizado en clave inclusiva.

Una educación en clave inclusiva supone la implementación de experiencias educativas situadas, vale decir, transformadas y adaptadas a las necesidades y requerimientos de los estudiantes (Onrubia, 2009), que desdibuje los principios de homogeneidad y estandarización educativa bajo los cuales son los propios estudiantes quienes deben adaptarse a la lógica del currículum (Essomba, 2006). En oposición a ello, y como forma de distribuir de manera equitativa las oportunidades de éxito educativo, la educación inclusiva privilegia la existencia de itinerarios heterogéneos y flexibles para el aprendizaje. De esta manera se espera que todo estudiante pueda acceder de manera igualitaria -pero no uniforme- a los aprendizajes esperados por la escuela.

Ahora bien, los itinerarios heterogéneos y flexibles de aprendizaje exhiben su máximo potencial cuando van de la mano con la entrega por parte del profesorado de ayudas educativas ajustadas, que tengan la capacidad de conectarse con las características y necesidades específicas de los estudiantes. Estas ayudas ajustadas permiten que cada estudiante construya su propia trayectoria de aprendizaje, siendo el profesorado el encargado de guiar este proceso hacia nuevas zonas de desarrollo (Besalú y Vila, 2007).

Aun cuando estas cuatro características centrales de la educación inclusiva se relacionan con una dimensión pedagógica de las prácticas educativas, no hay que olvidar que la inclusión contempla, además, un componente político que también se pone en juego en estas prácticas educativas (Pujolàs, 2012). La inclusión educativa contempla un componente político en la medida que busca un mayor grado de reconocimiento y participación de los diferentes grupos sociales, permitiendo que el currículum escolar sea más representativo de los diferentes referentes culturales del alumnado (Sleeter, 2015). De esta manera la inclusión no sólo busca ofrecer iguales oportunidades de aprendizaje para todos sus estudiantes, sino que visualiza la escuela como un espacio para igualar las posiciones de los diferentes grupos a los que pertenece el alumnado (Dubet, 2012), eliminando las desigualdades de aprendizaje con las que se encuentran algunos estudiantes de grupos minoritarios.

\subsection{El Modelo 5D}

Quinta Dimensión (5D) es un modelo de inclusión educativa desarrollado por Michael Cole en la década de los ochenta, en el contexto del Laboratorio de Cognición Humana Comparada de la Universidad de 
California (Cole, 1999). Su propósito original consistió en el diseño e implementación de sistemas de actividad que se constituyan en experiencias educativas de inclusión para aquellos participantes pertenecientes a grupos minoritarios. Si bien en su sentido original el modelo 5D fue desarrollado en entornos educativos no formales para la escolarización de colectivos históricamente segregados (Cole, 2006), en la actualidad se ha convertido en una red mundial de experiencias en diferentes entornos educativos. Fuertemente enraizado en una perspectiva histórico-cultural de entender los procesos de desarrollo y aprendizaje (Lamas y Lalueza, 2012) y de un enfoque culturalista de la psicología (Cole, 1999), las diferentes experiencias mundiales del modelo 5D se han constituido en auténticos laboratorios de prácticas para el desarrollo de experiencias educativas inclusivas y culturalmente significativas (Jiménez y Fardella, 2005). Aun cuando en la actualidad las diversas modalidades 5D varían en el diseño, las lógicas de funcionamiento, las propuestas metodológicas e incluso el grado de institucionalización, todas convergen en la importancia de generar experiencias educativas culturalmente relevantes, pedagógicamente significativas y socialmente justas.

Proyecto Trovadores. Este proyecto corresponde a una de las variantes que el modelo 5D ha asumido en el contexto español y que es implementado por el equipo DEHISI (Grupo de Investigación en Desarrollo Humano, Intervención Social e Interculturalidad) de la Universidad Autónoma de Barcelona. Este proyecto comenzó a implementarse en la década de los noventa en Cataluña, como una experiencia piloto para la escolarización de alumnado gitano en contextos de segregación y riesgo de exclusión social. En consonancia con los fundamentos 5D se presentó como forma de combatir, a través de experiencias educativas pertinentes y significativas, los altos índices de ausentismo, abandono y fracaso escolar en los que se veían envueltos dichos estudiantes. El lugar utilizado tradicionalmente por estudiantes gitanos es en la actualidad compartido por estudiantes inmigrantes que han enfrentado problemas escolares muy similares (Essomba, 2012). Actualmente el proyecto es implementado en seis entornos educativos: tres centros de primaria, dos asociaciones gitanas y un liceo localizados en el área metropolitana de Barcelona.

El proyecto Trovadores es un sistema de actividad con base en la creación de una comunidad de aprendizaje (Engeström, 2001). Esta comunidad se compone de equipos de trabajo que empleando el aprendizaje colaborativo crea narraciones digitales en torno a alguna temática definida libremente. Independientemente del tema elegido, el trabajo realizado por los grupos de estudiantes busca convertirse en una experiencia de aprendizaje significativo que logre conectarse con los conocimientos previos y fondos de conocimientos de los participantes y, al mismo tiempo, logre contemplar los intereses y motivaciones de sus integrantes (Ausubel, Novak y Hanesian, 1983; Coll, 1988).

A lo largo de un año académico y durante 90 minutos semanales, la tradicional asignatura de Tecnología se transforma en una comunidad de aprendizaje compuesta por los estudiantes de un curso. Liderada por el tutor y un coordinador del equipo de investigación DEHISI, más el soporte de un equipo de estudiantes en prácticas universitarias (Magisterio y Psicología), semana a semana equipos heterogéneos de trabajo ${ }^{1}$ van diseñando y dando forma a su proyecto digital. Conformación de equipos, conocimiento y cohesión grupal, distribución de roles y tareas, negociación y toma de decisiones, resolución de conflictos, diseño de un plan de trabajo, preparación de presentaciones en Power Point; confección de disfraces y escenografía, filmación y edición, así como presentación pública, son algunas de las tareas, procesos y experiencias que se van produciendo a modo de espiral a lo largo del proyecto y que constituyen parte central de su funcionamiento.

\section{Marco metodológico}

Con el objeto de describir y caracterizar las prácticas educativas desarrolladas en contextos multiculturales, y analizar en qué dimensiones de la práctica educativa y con qué implicancias la implementación del modelo 5D amplía las oportunidades de inclusión educativa en un contexto escolar con alta presencia de alumnado perteneciente a grupos minoritarios, se desarrolló una investigación etnográfica (Flick, 2009; Hammersley y Atkinson, 2005), compuesta de dos fases: la primera orientada a la

\footnotetext{
${ }^{1}$ Heterogéneos generacionalmente porque comparten tareas estudiantes de escuela con estudiantes universitarios; y heterogéneos culturalmente en la medida que los integrantes son catalanes, gitanos españoles, paquistaníes, indios, rumanos, bolivianos u hondureños, por citar algunas procedencias.
} 
caracterización de las prácticas educativas habituales y ordinarias, y la segunda a la caracterización de las prácticas educativas desarrolladas en el marco del proyecto Trovadores.

La principal justificación en la elección de este enfoque metodológico radica en que la etnografía escolar se constituye en un enfoque privilegiado para comprender un fenómeno de manera profunda (Rockwell, 2009) captando sus estructuras de significación (Velasco y Díaz de Rada, 2009), posibilidad que no otorgan otros enfoques que se mueven en capas más superficiales de las problemáticas de estudio (Geertz, 2003). Además, el sentido performativo de la etnografía escolar se constituye en un segundo argumento, en tanto una de las características distintivas de realizar estudios etnográficos ideográficos radica en su potencialidad para ofrecer nuevos datos, y nuevas perspectivas que contribuyan a la mejora de la realidad educativa estudiada (Serra, 2004). Dicho en otras palabras, la intencionalidad etnográfica no pasa únicamente por retratar una realidad determinada, sino justamente utilizar los datos producidos como plataforma de reflexión académica que decanten en propuestas de mejora para las propias realidades educativas participantes.

Dentro de las técnicas para la producción de datos se utilizó la observación participante (Spradley, 1980), herramienta que constituye la principal fuente de producción de datos de la etnografía escolar (Kawulich, 2005). Esto permite vivenciar -y no sólo observar- las prácticas educativas en su ambiente natural y de primera fuente, y producir una descripción sistemática de las prácticas estudiadas. El diario de campo se constituyó en un soporte central para la producción de datos y su posterior codificación y análisis.

Contexto y participantes. Del conjunto de experiencias de aplicación del proyecto (seis entornos educativos) se optó por focalizar la investigación en un centro de primaria en particular, debido a que era uno de los contextos con mayor presencia de alumnado de grupos minoritarios y por ubicarse en una zona de alto riesgo de exclusión social. La escuela es un Centro de Educación Infantil y Primaria, y al mismo tiempo un Centro de Atención Educativa Preferente. ${ }^{2}$ Se encuentra ubicado en la ciudad de Badalona (la tercera más grande de Cataluña y en la cual para el 2011 se contabilizaron poco más de 219,000 habitantes de acuerdo al registro de padrones municipales, ${ }^{3}$ de los cuales un $14.84 \%$ corresponde a población extranjera). Al analizar la composición demográfica de la población extranjera se observa que está compuesta por más de 75 nacionalidades. El barrio de San Roque, en el cual se encuentra la escuela, es uno de los tres barrios que concentran la mayor proporción de población inmigrante-de sus 14,000 habitantes, poco más de la mitad corresponde a familias gitanas.

Según información aportada por la propia escuela, para el año académico 2011-2012, período en el que se llevó a cabo la investigación, de un total de 366 estudiantes matriculados en el nivel infantil y primaria, un 36.34\% correspondía a estudiantes extranjeros (principalmente de África, América, Asia y Europa no comunitaria), un $47.81 \%$ a estudiantes de etnia gitana y un $15.85 \%$ a españoles de comunidades autónomas distintas de Cataluña. La prácticamente nula presencia de estudiantes autóctonos hace de esta escuela un auténtico ghetto escolar (Hannoun, 1992).

De los cuatro cursos participantes de esta escuela (las dos líneas de 50. y 60. sexto de primaria), se seleccionó un 6o. de primaria por tres razones fundamentales: 1) el curso presentaba una composición demográfica particularmente heterogénea en comparación a otros; 2) contaba con nuevos estudiantes con bajo nivel de dominio de la lengua vehicular, lo que hacía particularmente relevante observar las implicancias de intencionar un cambio en los sistemas de actividad educativos; y 3) el tutor contaba con varios años de experiencia en la implementación del proyecto, lo que implicaba un mayor grado de agencia por parte de la escuela en cuanto a la implementación del proyecto y menos intervención por parte del coordinador del grupo de investigación. Seleccionar este curso permitía, a fin de cuentas, observar una experiencia con un mayor grado de apropiación e institucionalización del proyecto (Lamas,

\footnotetext{
${ }^{2}$ De acuerdo a la orden del 3 de septiembre de 1996 de la Generalitat de Catalunya que cualifica a centros privados o públicos sostenidos con fondos públicos como preferentes, en la medida que corresponde a centros que se ubican en entornos sociales y económicos desfavorecidos, y que por tanto imparten la enseñanza a un número elevado de estudiantes que presentan dificultades para conseguir con éxito los objetivos educativos generales. Publicado en el DOGC, $n^{\circ} 2264,4 / 10 / 96$.

${ }^{3}$ Información disponible en www.idescat.cat
} 
2013). En el momento de la investigación el curso seleccionado estaba compuesto por 26 estudiantes, 14 mujeres y 12 hombres; en cuanto a su procedencia: 16 gitanos, 4 marroquíes, 2 bolivianos, 1 hondureño, 1 ruso, 1 indio y 1 paquistaní.

Trabajo de campo. La investigación consistió en realizar 20 acompañamientos de aula al maestro de 60. de primaria, tanto en las sesiones del proyecto Trovadores (10 sesiones) como en sus clases ordinarias (10 sesiones). En la medida que se consideró cada observación de aula como una sesión etnográfica (Wilcox, 1993), el análisis de datos se realizó sobre un total de 20 sesiones etnográficas. Una vez realizados los acompañamientos de aula se procedió a la transcripción, organización y sistematización de los datos para su posterior análisis.

Utilizando el software Atlas.ti (Muñoz, 2003) se seleccionaron y organizaron los datos a través de la creación de categorías emergentes que permitieron agrupar diferentes citas del corpus textual producido durante las sesiones etnográficas (Glaser y Strauss, 1967). Para establecer las categorías de la investigación se hizo una codificación abierta de todas aquellas citas, fragmentos y anotaciones temas de las notas de campo que aportaran información relevante de acuerdo a los objetivos, obteniendo así un primer nivel de organización del material (Holton, 2010).

Con la participación de tres especialistas en el ámbito de la inclusión escolar en contextos de diversidad cultural se realizó una validación interjueces del primer listado de códigos emergentes, seleccionando aquellos que presentaran un mayor nivel de consistencia interna y robustez teórica. Рara lograr esto se entregó a cada uno de los investigadores un listado inicial de códigos (33) con sus respectivas definiciones, así como un corpus que previamente había sido categorizado con dicho código, como parte del primer análisis. Al comparar la codificación inicial con la codificación realizada por los pares expertos se pudo establecer la consistencia de los códigos. Esto significa que un mismo texto o cita había sido codificado por los investigadores y los jueces con la misma categoría, con lo cual había coincidencia en su uso. Esta secuencia de trabajo permitió seleccionar finalmente nueve códigos en los que hubo más de un $75 \%$ de coherencia en la validación interjueces, es decir, categorías que fueron utilizadas por los distintos investigadores para codificar las mismas citas del corpus. Estos nueve códigos corresponden a los que finalmente se utilizaron para organizar y analizar los datos producidos.

\section{Resultados}

En virtud de que un análisis exhaustivo de todos los códigos escapa a las posibilidades de este trabajo, se exponen los hallazgos que guardan una mayor relación con los referentes conceptuales presentados anteriormente. De esta manera, de los nueve códigos construidos se presentarán los resultados de tres: Vinculación (CV), Participación (PP) y entrega de Ayudas (CA). Para caracterizarlos se utilizan fragmentos de las notas de campo realizadas durante las observaciones de aula.

Se han organizado los resultados de manera diferenciada para cada contexto de actividad (aula tradicional y proyecto Trovadores) de manera que se tenga una mirada global y comprensiva sobre cada uno, reservando para el espacio discusión el establecimiento de relaciones y tensiones entre los resultados obtenidos.

\subsection{Los imponderables del aula tradicional}

En relación con la vinculación (cv) de los contenidos curriculares con las experiencias previas y los referentes culturales del alumnado, predomina durante las clases una escasa intencionalidad del profesor por establecer continuidad (entre los referentes culturales y el patrimonio familiar del alumnado) con los contenidos abordados y las actividades realizadas. Lo recurrente durante las clases son actividades en las que quedan en evidencia las dificultades que enfrentan algunos estudiantes para otorgar significatividad psicológica y cultural a los contenidos vistos. Esto ocurre en la medida en que al considerar la composición cultural de los estudiantes, muchos contenidos curriculares abordados son percibidos como temas lejanos a los que no resulta fácil atribuir un significado. 
Voy llegando a la escuela, se me acerca Mateo ${ }^{4}$ (alumno gitano) y me dice que hoy no habrá proyecto Trovadores, ya que han terminado la semana pasada. Este comentario me muestra que él claramente relaciona mi presencia en la escuela con la actividad. Le pregunto entonces qué harán en vez del proyecto y me explica que tendrán clases de plástica: "Cosas de esto", agrega, mostrándome unas figuras de yeso. Concluye su explicación diciendo "perder el tiempo". Una vez hemos subido y nos ubicamos en el aula, el profesor Marco da las instrucciones de la actividad que estará enfocada en la comprensión oral y escrita del catalán; señala que trabajarán con la música de una canción catalana muy famosa y que cada uno deberá trabajar en ficha, rellenando aquellas palabras que faltan para completar la letra de la canción. Observo que hay una especie de desánimo general y de aburrimiento. La mayoría de los estudiantes sigue con poco interés la actividad y prefieren conversar entre ellos o hacer otras cosas personales. Sigue la música y veo que Danial (alumno indio), quien presenta un bajo dominio del catalán y español, está en primera fila detrás de la mesa del profesor pero se dedica a jugar con los materiales que están sobre ella. No se necesitan muchos elementos para darse cuenta que no entiende nada de la canción y que muy probablemente no comprendió ni las instrucciones. [OA_BS_6a_26.11.10]

Esta escena es representativa del funcionamiento general de las clases observadas, en las que los procesos de enseñanza y aprendizaje no sólo reflejan la falta de sensibilidad cultural en la elección de los contenidos a abordar, sino que ejemplifica la actitud de poca relevancia y motivación que este tipo de actividades pedagógicas provoca entre el alumnado. Como se ha planteado anteriormente, los aprendizajes se producen en la medida que los estudiantes internalizan y se apropian del conocimiento, proceso que requiere que se atribuya un sentido a dicha internalización. Así, la escena anterior evidencia que la falta de sentido y pertinencia cultural de las actividades contribuye a disminuir las oportunidades de aprendizaje de aquellos estudiantes de grupos sociales minoritarios. La vinculación entre contenidos y experiencia cultural se constituye en un componente indispensable para garantizar el aprendizaje.

Desde el punto de vista de la participación (PP), cabe mencionar situaciones y aspectos que guardan relación con las formas en que los participantes se relacionan entre sí (formas de relación directamente condicionadas por la estructura de la tareas académicas) es recurrente en las clases una dinámica en la que existen muy pocas instancias de interacción entre el alumnado, ya que la mayoría de las actividades desarrolladas se llevan a cabo de forma individual, modalidad que restringe las oportunidades de colaborar en actividades orientadas a metas comunes y de compartir recursos para desarrollar aprendizajes.

Comienzan a repartirse las hojas de trabajo por lo que también llega una a mis manos. Observo las que tienen los estudiantes y compruebo que es la misma para todos, no está dividida por dificultad o con algunas ayudas para algunos. Todos realizarán el mismo ejercicio bajo las mismas condiciones. Compruebo por lo demás que es una actividad que se realizará individualmente. (OA_BS_6a_26.11.10_1)

Marco explica la actividad de la clase de hoy que consiste en cómo buscar palabras en un diccionario: "Algo que algunos chicos no saben y es importante para cuando vayan al instituto", señala después. Luego de buscarlas hay que anotar en la libreta el significado de cada una de ellas. Presentada la actividad, Juan Raimundo (alumno gitano) pregunta al profesor si el trabajo de las palabras se puede hacer de más de uno, pero él dice que no argumentando "quiero que aprendas tú solo", a lo que Juan Raimundo responde que ya lo sabe, por lo que finalmente Marco le dice "demuéstramelo". (OA_BS_6a_06.06.11_9)

Este énfasis en el trabajo autónomo no necesariamente es conciente de las dificultades que para ciertos estudiantes supone la realización de las actividades, ubicando a los estudiantes minoritarios en condiciones de desigualdad con relación al éxito académico debido a sus dificultades en el manejo de la lengua vehicular o al escaso dominio de los referentes del currículum hegemónico.

\footnotetext{
${ }^{4}$ Los nombres en las transcripciones han sido modificados para proteger el anonimato y la confidencialidad de los participantes.
} 
Ahora bien, en los casos en que las actividades permiten el trabajo grupal, se integran equipos que según las observaciones realizadas- evidencian al menos dos formas principales: el primero en función del género y el segundo de ellos en función de la pertenencia étnica.

Termina la actividad de trabajo individual en torno a un dossier y Marco pide a los estudiantes que cambien de lugar, que cada uno se ponga donde quiera. Esto hace que gran parte de los estudiantes se agrupen, fundamentalmente, en función de sus afinidades. Hay excepciones como Faisan (alumno árabe) que mira el resto de las mesas pero no sabe dónde ubicarse, por lo que finalmente se queda donde mismo, es decir, junto a Mateo (alumno gitano). Nabila y Wania (alumnas gitanas) aprovechan para juntarse. Alexandra y Vannesa (alumnas latinas) permanecen en la misma mesa junto a Maicon (alumno hondureño) que escoge esta mesa y Gerson (alumno boliviano) que estaba anteriormente también conformaba una mesa de latinos. Llama la atención que Francüsca y Danial (alumna rusa y alumno paquistaní) hayan estado toda la hora solos, sin tener ningún contacto con sus compañeros. (OA_BS_6a_17.05.11_6).

La poca planificación de los agrupamientos por parte del profesor hace que los estudiantes que encuentran mayores barreras para la participación, no logren incluirse y terminen trabajando de manera individual, en algunos casos llegando a situaciones de patente aislamiento y, por tanto, exclusión escolar. De esta manera, lo que se observa a partir de los agrupamientos es que nuevamente es un recurso pedagógico que beneficia a una parte del alumnado, dejando en situación de desventaja educativa a aquellos que por diversas razones no logran incluirse ni participar en los grupos de trabajo formados de forma espontánea.

Por último, y en estrecha vinculación con las formas predominantes de participación, respecto a la entrega de ayudas (CA), es posible observar que éstas son limitadas, distribuidas sin un criterio claro y no siempre ofrecidas de manera oportuna a los requerimientos de los estudiantes. Pese a que se evidenciaron casos de una suerte de "apadrinamiento" por parte del profesor hacia determinados estudiantes con mayores dificultades para seguir con éxito las actividades pedagógicas, lo cierto es que son más significativas las situaciones en las que una parte del alumnado permanece aislada y desconectada de estas actividades.

Luego de un tiempo de trabajo individual en torno a la realización de una receta culinaria, el profesor le pide a Faisan (alumno paquistaní), llegado hace muy poco tiempo al país y la escuela, que lea en voz alta su trabajo. Lo hace en voz muy baja por lo que el profesor le pide que repita la lectura. Algunos compañeros se muestran confundidos y preguntan qué es lo que ha hecho. Él vuelve a leer su trabajo que es un listado de platos de su país (kebab, durum) El resto de los compañeros comienza a comentar en voz alta que Faisan se ha equivocado. El profesor explica al grupo que Faisan se ha equivocado, que ha hecho un listado de platos cuando lo solicitado era hacer una receta. Finalmente el profesor se dirige al alumno diciéndole "No pasa nada", como aceptando su equivocación. Luego explica en voz alta que esta equivocación se ha producido porque hoy no ha venido Mohamad (alumno paquistaní) que es quien comúnmente le explica en su idioma las actividades que hay que realizar. [OA_BS_6b_06.06.11].

De este fragmento se puede observar la importancia y lo decisivo que puede ser una entrega oportuna y ajustada de ayudas, que permita a los estudiantes que lo requieran contar con un mayor grado de andamiaje en la realización de la actividad. Estas ayudas no sólo permitirían orientar de mejor manera el proceso de enseñanza y aprendizaje de estos estudiantes, sino además permitir a éstos adquirir el control en las tareas y lograr mayores niveles de autonomía en el aprendizaje.

\subsection{Los imponderables del proyecto Trovadores}

Respecto a la vinculación (cv) se observa que el proyecto configura un sistema de actividad educativa que aumenta significativamente el hecho de que los fondos de conocimientos y el patrimonio cultural del alumnado puedan ser incorporados a los procesos de enseñanza y aprendizaje. En la medida que el alumnado asume un mayor grado de control en la toma de decisiones, recurre con mayor frecuencia a tomar elementos propios de sus diversas culturas y a utilizarlos, en consecuencia, como materia prima 
para los aprendizajes. Adicionalmente, se ha constatado que esta mayor sensibilidad cultural del proyecto permite que el alumnado pueda darle un sentido y significado a las actividades educativas, apropiándose tanto de sus objetivos y herramientas utilizadas como de las metas perseguidas.

Durante las primeras semanas de inicio del proyecto, los estudiantes ya han conformado grupos de trabajo y en esta sesión trabajan en parejas en la elaboración de sus presentaciones personales primero a través de una descripción escrita y luego en versión digital en Power Point. La mayoría de las presentaciones están listas en cuanto al diseño escogido por cada estudiante, faltando sólo que cada uno de agregue una pequeña biografía que los describa junto a la foto personal. Voy viendo el trabajo de distintas parejas, leyendo las descripciones que hay de cada uno y reparo en que es una oportunidad para que todos puedan visibilizar y compartir con el resto del curso algunos elementos culturales como la procedencia, algunos referentes familiares como la religión así como los gustos personales por la música, el baile o los deportes. Es así como en las presentaciones aparecen cantaores gitanos, salteñas bolivianas, nombres en lenguas originarias y vestimentas árabes como el hiyab. Si bien la actividad podría explotar aún más este recurso, es un buen punto de partida que cuando se realicen las presentaciones grupales, se visibilice la diversidad cultural del grupo y cada uno de los estudiantes tenga la oportunidad de dar a conocer aspectos de su biografía cultural considerados relevantes. [T_BS_6a_30_28.09.11].

Este fragmento demuestra cómo el desarrollo de ciertos objetivos pedagógicos como son en esta caso la expresión escrita en la lengua vehicular, así como el desarrollo de competencias en el manejo de las TIc, pueden trabajarse en estrecha relación a temáticas y contenidos de interés y relevancia para los estudiantes. Esto aumenta significativamente el interés en la tarea académica y permite a todos los estudiantes lograr con éxito los aprendizajes esperados.

En relación con la participación (PP) el proyecto Trovadores no sólo la promueve sino que la requiere: el logro de las metas y el avance grupal está supeditado a que los participantes se involucren en las distintas tareas y cumplan con los objetivos planteados. De esta manera, no sólo es necesario que los estudiantes adopten un rol activo en este sistema de aprendizaje, sino que es inherente a la actividad que dicha participación adopte un formato colaborativo, con lo cual el éxito de las diferentes actividades (diseñar un Power Point de presentación grupal, ensayar una coreografía, filmar o editar entrevistas a vecinos del barrio) se logra a partir de la interdependencia y el trabajo de todos. Esta participación plena permite que las oportunidades de aprendizaje se distribuyan de forma equitativa para todos sus participantes, evitando que las prácticas educativas monopolicen las oportunidades de participación en un grupo de estudiantes.

En el grupo que conduce Marta (practicante de Psicología) hay dos integrantes que habitualmente no participan en clases: Yasser, alumno paquistaní con bajo dominio de la lengua y Jerson, alumno latino de actitud introvertida. Si bien durante las primeras sesiones la tendencia era que el trabajo y las decisiones se concentran en los estudiantes gitanos que también componen el grupo, Marta se ha encargado de permitir que todos opinen, recurriendo incluso a la traducción de otro estudiante paquistaní para que Yasser pueda opinar. Han pasado varias semanas de esta nueva modalidad y se nota cómo el grupo ha logrado hacer una distribución equitativa del trabajo, permitiendo que algunos trabajen en el computador, otros en el diseño de disfraces, en la sistematización de las decisiones tomadas y otro actuando como portavoz ante el maestro. Aun cuando se sigue evidenciando un desbalance en el número de tareas, todos han estado contribuyendo desde sus posibilidades para hacer avanzar el proyecto que han decidido realizar. [T_BS_6a_10﹎23.11.11].

Finalmente y con relación a la entrega de ayudas (CA), el hecho que la actividad rompa la lógica habitual dando paso al trabajo en pequeños equipos, amplifica las oportunidades tanto del maestro como de los propios compañeros de atender a las necesidades de los estudiantes que presentan mayores dificultades. Estas ayudas adicionales y ajustadas permiten que todos puedan avanzar en igualdad de condiciones hacia el desarrollo de las competencias que el proyecto moviliza. 
Me acerco a ver cómo está trabajando dentro de un grupo una pareja de estudiantes paquistaníes en la que hay un alumno que se ha incorporado hace un par de meses al curso. Se observa cómo Mohamad, alumno antiguo, utiliza el urdu para explicarle qué hay que hacer y en qué tareas puede colaborar, puesto que no comprende el castellano. Es una acción muy potente, ya que se aprovecha la diversidad lingüística para que todos puedan participar de la actividad. El profesor ha tenido en cuenta este criterio a la hora de armar los grupos, privilegiando esta suerte de "apadrinamientos" que son habituales en el proyecto pero que no se ven de manera consolidada en las clases normales. [OA_BS_6a_26.11.10_4].

El trabajo en grupos pequeños permite además que el propio profesor vaya viendo el trabajo de cada grupo en particular y al mismo tiempo vaya monitoreando el desempeño de ciertos estudiantes con mayores dificultades para el aprendizaje. En aquellos casos en los que identifica la necesidad de una mayor intervención, se incorpora al trabajo del grupo o bien realiza algunas modificaciones en el funcionamiento grupal permitiendo que algunos participantes reciban apoyos adicionales.

Continúa el trabajo en parejas haciendo las respectivas presentaciones en Power Point. Tanto la coordinadora de la actividad como el profesor aprovechan esta modalidad de trabajo para ir por las parejas aclarando dudas, ayudando a resolver dificultades y atender a las solicitudes de los estudiantes. Esto se hace en un ambiente de mucha horizontalidad y de manera muy dialógica: preguntan, dan pistas y permiten de esta forma que sean los propios estudiantes quienes lleguen a encontrar las soluciones. En aquellos casos de estudiantes con mayores dificultades, se sientan a trabajar con ellos y hacen un trabajo más personalizado y pausado hasta que los estudiantes logran adquirir mayor autonomía en las tareas que están realizando. La coordinadora también se mueve por los grupos revisando las biografías personales pero su interés está centrado en verificar si se está utilizando bien el catalán. Cuando detecta errores, o bien los corrige o hace preguntas para que los propios estudiantes descubran los errores. Algo similar hacer al preguntarle a Samuel (alumno gitano) por la elección de un color favorito, estimulándolo a pensar mejor por qué lo ha escogido, qué representa ese color para él o en qué elementos del barrio podría encontrar ese color. [T_BS_6a_30_28.09.11].

\section{Discusión}

Como el trabajo etnográfico realizado evidencia, las implicancias pedagógicas de las prácticas educativas pueden llegar a ser significativamente distintas, en función de los principios que guíen y organicen los sistemas de actividad de los cuales éstas formen parte. Asumiendo que la significatividad de los aprendizajes depende de su contexto de producción (Cole, 1999) más que de características internas de los aprendices, queda en evidencia que cuando las prácticas educativas logran incorporar el background de sus participantes, cuando las oportunidades de participación se distribuyen de manera equitativa y cuando los estudiantes reciben ayudas ajustadas a sus necesidades, el sistema de actividad se vuelve más inclusivo (Ainscow, 2012).

En ese sentido es posible observar que cuando las experiencias educativas permiten establecer continuidades entre el currículum escolar y los conocimientos previos y las experiencias culturales de los estudiantes, aumenta la significatividad lógica, psicológica y cultural de éstas de dichas experiencias (Cochran-Smith, 2004). Este aumento de significatividad se refleja en un mayor compromiso y motivación por parte de los estudiantes en participar y apropiarse de las experiencias educativas de las que forman parte. Establecer este tipo de continuidades hace que las actividades sean desde el punto de vista pedagógico más sensibles culturalmente hablando, permitiendo resolver uno de los principales problemas de la escolarización en contextos multiculturales, como es la poca significatividad y relevancia de su oferta educativa (Palaudàrias y Garreta, 2008; Pàmies, 2011). Sin embargo, un mayor reconocimiento de las experiencias y el conocimiento de los estudiantes de grupos desfavorecidos no contribuye únicamente al desarrollo de aprendizajes significativos; la oportunidad de traer al espacio escolar referentes culturales se constituye en un principio fundamental de justicia curricular (Connell, 1997), en tanto da espacio para el desarrollo de un currículum inclusivo que incorpora las perspectivas de los distintos estudiantes que conforman el grupo curso. A diferencia de lo que ocurre habitualmente 
desde lógicas educativas asimilacionistas y compensatorias, los antecedentes culturales y lingüísticos en lugar de ser percibidos como un obstáculo o algo problemático y deficitario que se debe superar, se convierten en el punto de partida para generar y promover logros académicos (Van Olphen y Ríos, 2004).

Se ha podido documentar además que cuando las interacciones se reorientan hacia formas colaborativas de trabajo, aumentan los espacios de participación individual y cultural, se evitan las experiencias de exclusión educativa, al tiempo que se multiplican las oportunidades del profesorado contactarse de manera más directa y oportuna con las necesidades de todos sus estudiantes. Esta nueva configuración favorece por tanto vínculo pedagógico profesor-estudiante que permite el despliegue de ayudas más ajustadas a dichas necesidades y la entrega de mayores oportunidades para el aprendizaje (Carrasco, Pàmies y Narciso, 2012).

Como se ha planteado al comienzo de este trabajo, las dificultades que presenta una parte de los estudiantes para seguir y apropiarse con normalidad de las experiencias educativas no obedecen necesariamente a características personales de éstos y no se explican por tanto por teorías del déficit cultural (Jenks, Lee y Kanpol, 2001; Sleeter y Grant, 2009), sino que se explican por la existencia de barreras para el aprendizaje y la participación que las propias experiencias educativas (y el profesorado) construye. En ese sentido una mayor pertinencia de los contenidos de aprendizaje, mayores grados de participación e interacciones colaborativas y mayor disponibilidad de ayudas para el aprendizaje se constituyen en tres cambios del sistema de actividad educativo que constituyen prácticas inclusivas (Booth y Ainscow, 2011).

\section{Reflexiones finales}

Más que hacer una apología del modelo 5D, esta investigación evidencia que pese a la abundante literatura que respalda la necesidad de formar y capacitar al profesorado para el desarrollo de prácticas educativas inclusivas e interculturales (Aguado, Gil y Mata, 2008; Essomba, 2006; Jordán, 2000), cambios sutiles en la forma de diseñar dichas prácticas tienen la potencialidad para incrementar las oportunidades de éxito educativo del alumnado perteneciente a minorías étnicas y culturales. El desafío pasa más bien porque estos cambios no se constituyan en un patrimonio del proyecto, sino que tengan la capacidad de irradiarse a otros espacios escolares. Este proceso de irradiación permitiría avanzar desde el desarrollo exclusivo de prácticas inclusivas al desarrollo de culturas escolares inclusivas (Booth y Ainscow, 2011), haciendo de la inclusión algo que vaya más allá de iniciativas puntuales y le otorgue un carácter de proceso en constante movimiento y reformulación (Echeita y Sandoval, 2002). Crear culturas escolares inclusivas en contextos multiculturales apuntaría a la creación de una comunidad educativa orquestada por valores que apuesten por el desarrollo de experiencias educativas significativas y de calidad para todos sus participantes.

\section{Referencias}

Aguado, T., Gil, I. y Mata, P. (2008). El enfoque intercultural en la formación del profesorado. Revista Complutense de Educación, 19(2), 275-292.

Ainscow, M. (2013). From special education to effective schools for all. En L. Florian, The sage handbook of special education. Londres: Sage.

Ainscow, M. (2012). Haciendo que las escuelas sean más inclusivas: lecciones a partir del análisis de la investigación internacional. Revista de Educación Inclusiva, 5(1), 39-49.

Ausubel, D., Novak, J. y Hanesian, H. (1983). Psicología educativa. Un punto de vista cognoscitivo. México: Trillas.

Crespo, I., Lalueza, J. L., Portell, M. y Sanchez, S. (2005). Communities for intercultural education: Interweaving microcultures. En M. Nilsson, y H. Nocon, School of tomorrow: teaching and technology in 
local and global communities. Berna: Peter Lang.

Banks, J. A. (1989). Approaches to Multicultural Curriculum Reform. Trotter Review, 3(3).

Besalú, X. (2010). Formar ciutadans i ciutadanes per a una catalunya plural, lliure, desacomplexada i justa. Valors, Idees, Actituds, 13, 119-132.

Besalú, X. y Vila, I. (2007). La buena educación: libertad e igualdad en la escuela del siglo XXI. Madrid: Los Libros de la Catarata.

Booth, T. y Ainscow, M. (2011). Index for inclusión: developing learning and participation in schools. Bristol: Centre for Studies on Inclusive Education.

Bronfenbrenner, U. (1987). La ecología del desarrollo humano. Barcelona: Paidós.

Carrasco, S., Pámies, J. y Narciso, L. (2012). A propósito de la acogida de alumnado extranjero. Paradojas de la educación inclusiva en Cataluña. Revista Latinoamericana de Educación Inclusiva , 6(1), 105-122.

Cochran-Smith, M. (2004). Walking the road: race, diversity and social justice in teacher education. Nueva York: Teachers College Press.

Cochran-Smith, M., Ludlow, L., Ell, F., O'Leary, M. y Enterline, S. (2012). Learning to teach for social justice as a cross cultural concept: findings from three countries. European Journal of Education Research, 1(2), 171-198.

Cole, M. (2006). The fifth dimension: an after-school program built on diversity. Nueva York: Russell Sage Foundation.

Cole, M. (1999). Psicología Cultural. Madrid: Morata.

Coll, C. (1988). Significado y sentido en el aprendizaje escolar. Reflexiones en torno al concepto de aprendizaje significativo. Infancia y Aprendizaje, 41, 131-142.

Connell, R. (1997). Escuelas y justicia social. Madrid: Morata.

Dubet, F. (2012). Los límites de la igualdad de oportunidades. Revista Nueva Sociedad, 239, 42-50.

Echeita, G. y Sandoval, M. (2002). Educación inclusiva o educación sin exclusiones. Revista de Educación, $327,31-48$

Engeström, Y. (2001). Expansive learning at work: toward an activity-theoretical reconceptualitation. Journal of Education and Work, 14(1), 133-156.

Essomba, M. (2012). Inmigración, sociedad y educación en la UE. Hacia una política educativa de plena inclusión. Cultura y Educación, 24(2), 137-148.

Essomba, M. (2006). Liderar escuelas interculturales e inclusivas. Equipos directivos y profesorado ante la diversidad cultural y la inmigración. Barcelona: Graó.

Esteban-Guitart, M. y Moll, L. (2014). Funds of identity. A new concept based on funds of knowledge approach. Culture y Psychology, 20, 31-48.

Esteban-Guitart, M., Oller, J. y Vila, I. (2012). Vinculando escuela, familia y comunidad a través de los fondos de conocimiento e identidad. Un estudio de caso con una familia de origen marroquí. Revista de Investigación Educativa, 10(2), 21-34. 
Flick, U. (2009). An introduction to qualitative research. Londres: Sage.

Garreta, J. (2011). La atención a la diversidad cultural en cataluña: exclusión, segregación e interculturalidad. Revista de Educación, 355, 213-233.

Glaser, B. y Strauss, A. (1967). Discovery of grounded theory. Chicago: Aldine.

Geertz, C. (2003). La interpretación de las culturas. Barcelona: Gedisa.

Hammersley, M. y Atkinson, P. (2005). Etnografía. Métodos de investigación. Barcelona: Paidós.

Hannoun, H. (1992). Els ghettos de l'escola. Per una educació intercultural. Recuperado de

http://experienciesdungruplector.blogspot.es/1399411232/els-ghettos-de-l-escola-per-una-educaciomulticultural//

Holton, J. (2010). The coding process and its challenges. Grounded Theory Review, 9(1), 21-40.

Jenks, C., Lee, J. y Kanpol, B. (2001). approaches to multicultural education in preservice teacher education: philosophical frameworks and models for teaching. The Urban Review, 33(2), 87-105.

Jiménez, F. y Fardella, K. (2015). Diversidad y rol de la escuela: discursos del profesorado en contextos educativos multiculturales en clave migratoria. Revista Mexicana de Investigación Educativa, 20(65), 419441. Recuperado de http://www.comie.org.mx/v1/revista/portal.php?idm=es\&sec=SC03\&\&sub=SBB\&criterio=ART65004

Jordán, J. A. (2000). Influencia del profesorado en la integración escolar del alumnado minoritario. Teoría de la Educación, 12, 67-84.

Kawulich, B. (2005). La observación participante como método de recolección de datos. Forum: Qualitative Social Research, 6(2).

Lalueza, J. L. (2012). Modelos psicológicos para la explicación de la diversidad cultural. Cultura y Educación, 24(2), 149-162. Recuperado de

http://www.tandfonline.com/doi/abs/10.1174/113564012804932119

Lamas, M. (2013). Innovación escolar: un análisis histórico-cultural y sistémico de cambio (Tesis doctoral). Universidad Autónoma de Barcelona.

Lamas, L. y Lalueza, J. L. (2012). Apropiación de un modelo colaborativo en escuelas multiculturales con alumnado en situación de exclusión social. Cultura y Educación , 24(2), 177-191. Recuperado de http://www.tandfonline.com/doi/abs/10.1174/113564012804932083

Lawrence-Brown, D. y Sapon-Shevin, M. (2014). Condition critical. Key principles for equitable and inclusive education. Nueva York: Columbia University.

Lazar, A. (2013). Degrees toward social justice teaching: examining the dispositions of three urban earlycareer teachers. The Urban Review, 45(5), 701-727

Muñoz, J. (2003). Análisis cualitativo de datos textuales con ATLAS/ti. Universitat Autònoma de Barcelona: Recuperado de http://www.ugr.es/ textinfor/documentos/manualatlas.pdf

OECD (2013). PISA 2012 results. Excellence through equity-giving every student the chance to succeed (Vol. II). Autor. 
Ogbu, J. (2005). Etnografía escolar. Una aproximación a nivel múltiple. En A. Díaz, H. Velasco y F. García (Eds.), Lecturas de antropología para educadores. El ámbito de la antropología de la educación y de la etnografía escolar (pp. 145-174). Madrid: Trotta.

Onrubia, J. (2009) Transformar para adaptar, adaptar para incluir: una mirada psicoeducativa a la educación inclusiva. En C. Giné (Coord), La educación inclusiva. De la exclusión a la plena participación de todo el alumnado. Barcelona: Horsori.

Palaudàrias, J. y Garreta, J. (2008). La acogida del alumnado de origen inmigrante: un análisis comparado desde la situación en Cataluña. Revista Española de Educación Comparada, 14, 49-78.

Pàmies, J. (2011). Las identidades escolares y sociales de los jovenes marroquíes en Cataluña. Psicoperspectivas. Individuo y sociedad, 10(1), 144-168.

Poveda, D. (2003). Saberes sociolinguísticos en una clase multicultural. En D. Poveda, Entre la diferencia y el conflicto. Miradas etnográficas a la diversidad cultural en educación (pp. 67-98). Cuenca: Universidad de Castilla-La Mancha.

Pujolàs, P. (2012). Aulas inclusivas y aprendizaje cooperativo, Educatio Siglo XXI, 30(1), 89-112

Rockwell, E. (2009). La experiencia etnográfica. Historia y cultura en los procesos educativos. Buenos Aires: Paidós.

Serra, C. (2004). Etnografía escolar, etnografía de la educación. Revista de Educación, 334, 165-176.

Sleeter, C. (2015). Teaching for social justice in multicultural classrooms. Multicultural Education Review, $5(2), 1-18$.

Sleeter, C. y Grant, C. (2009). Making choices for multicultural education: five approaches to race, class and gender. Hoboken, NJ: Wiley.

Spradley, J. (1980). Participant observation. Nueva York: Holt, Rinehart and Winston.

Van Holphen, M. y Ríos, F. (2004). Al servicio de la justicia social: conocimientos, destrezas y actitudes para los nuevos tiempos. Psicoperspectivas, 3, 151-171.

Velasco, H. y Díaz, A. (2009). La lógica de la investigación etnográfica. Un modelo de trabajo para etnógrafos de escuela. Madrid: Trotta.

Wilcox, K. (1993). La etnografía como una metodología y su aplicación al estudio de la escuela: una revisión. En A. Díaz, H. Velasco y J. García (Eds.), Lecturas de antropología para educadores. El ámbito de la antropología de la educación y de la etnografía escolar (pp. 95-126). Madrid: Trotta. 